\title{
Blow up solutions for a Liouville equation with singular data*
}

\author{
Pierpaolo Esposito ${ }^{\dagger}$
}

\begin{abstract}
We consider the asymptotic analysis and some existence result on blowing up solutions for a semilinear elliptic equation in dimension 2 with nonlinear exponential term, singular sources and Dirichlet boundary condition.
\end{abstract}

\section{Introduction}

We are concerned with the study of a class of two-dimensional semilinear elliptic problems with exponential nonlinearity and possibly singular data given by Dirac masses.

The regular problem, when no singular data is present, is of interest in conformal geometry [Au], statistical mechanics [CLMP1]-[CLMP2] and several other areas of applied mathematics, and has been widely investigated in the past years.

While, the singular problem has been considered more recently, as it naturally arises in the study of vortices in various selfdual Gauge field theories, see [BBH], [Ya] and references therein.

In all those contexts, there is a definite interest to construct solutions which "blow up" and "concentrate" at a set of given points, whose location carries relevant information about the geometri$\mathrm{cal} /$ physical properties of the problem under exam.

\section{$2 \quad$ A physical model}

To motivate the relevance of singular Liouville problems, we present the abelian Chern-Simons model, which is a planar theory introduced in the '90 to describe interesting phenomena in anyonic particle physics such as the high critical temperature superconductivity and the quantum Hall effect. The Higgs-Chern-Simons theory, proposed by Hong-Kim-Pac [HKP] and Jackiw-Weinberger [JW], is described by the Lagrangean density

$$
\mathcal{L}(\mathcal{A}, \phi)=-\frac{k}{4} \varepsilon^{\alpha \beta \gamma} A_{\alpha} F_{\beta \gamma}+D_{\alpha} \phi \overline{D^{\alpha} \phi}-\frac{1}{k^{2}}|\phi|^{2}\left(|\phi|^{2}-1\right)^{2},
$$

where the (dimensionless) coupling constant $k$ is known as the Chern-Simons parameter and $\left\{\varepsilon^{\alpha \beta \gamma}\right\}$ is the totally antisymmetric tensor with $\varepsilon^{012}=1$. The variables $\mathcal{A}, \phi$ are defined over the Minkowski space $\left(\mathbb{R}^{1+2}, g\right)$ and the metric tensor $g=\operatorname{diag}(1,-1,-1)$ is used to lower and raise indices.

The potential field $\mathcal{A}=-i \sum_{\alpha=0}^{2} A_{\alpha} d x^{\alpha}$, a 1-form, is specified by the components: $A_{\alpha}: \mathbb{R}^{1+2} \rightarrow \mathbb{R}$,

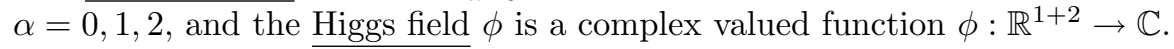

The Maxwell gauge field $F_{\mathcal{A}}=-\frac{i}{2} \sum_{\alpha, \beta=0}^{2} F_{\alpha \beta} d x^{\alpha} \wedge d x^{\beta}$, a 2-form, is specified by the components:

$$
F_{\alpha \beta}=\partial_{\alpha} A_{\beta}-\partial_{\beta} A_{\alpha} .
$$

\footnotetext{
*Supported by M.U.R.S.T., project "Variational methods and nonlinear differential equations".

†Dipartimento di Matematica, Università degli Studi "Roma Tre", Largo S. Leonardo Murialdo, 1 - 00146 Roma, e-mail esposito@mat.uniroma3.it.
} 
The covariant derivative $D_{A}$ weakly couples the Higgs field $\phi$ with the potential field $\mathcal{A}$ :

$$
D_{A} \phi=\sum_{\alpha=0}^{2} D_{\alpha} \phi d x^{\alpha}, \quad D_{\alpha} \phi=\partial_{\alpha} \phi-i A_{\alpha} \phi .
$$

Note that the gauge field $F_{\mathcal{A}}$ can be seen as the curvature associated to the connection $\mathcal{A}$.

In superconductivity, $\phi$ plays the role of an ordering parameter, and so $|\phi|$ measures the number density of the (superconductive) Cooper pairs. So, where $\phi=0$ we have a normal state, while $|\phi|>0$ gives a mixed state and $|\phi|=1$ (in this normalization of constants) gives a perfect superconductive state.

The Gauge invariance of the theory is given by the tranformation:

$$
\left\{\begin{array}{l}
\phi \rightarrow e^{i \omega} \phi \\
\mathcal{A} \rightarrow \mathcal{A}+d \omega
\end{array}\right.
$$

for any real function $\omega$ over $\mathbb{R}^{1+2}$. Notice that, although the Lagrangean density is not invariant under such Gauge transformations, the associated second-order Euler-Lagrange equations do have such an invariance.

The Euler-Lagrange equations are very difficult to handle analitically, but fortunately the structure of $\mathcal{L}$ allows a selfdual first-order factorization which in the static case takes the following form:

$$
\begin{array}{cc}
D_{+} \phi:=\left(\partial_{1}+i \partial_{2}\right) \phi-i\left(A_{1}+i A_{2}\right) \phi=0 & \\
F_{12}=+\frac{2}{k^{2}}|\phi|^{2}\left(1-|\phi|^{2}\right) & \\
2 A_{0}|\phi|^{2}=k F_{12} & \text { (Gauss law). }
\end{array}
$$

Equation (1) is a Gauge invariant version of the Cauchy-Riemann equation and implies holomorphictype properties for the Higgs field $\phi$. In particular, we see that $\phi$ admits a finite number of zeroes $p_{1}, \ldots, p_{N}$ with integral multiplicities $\alpha_{1}, \ldots, \alpha_{N}$, which are known as the vortex points and therefore represent defects of the superconductive state. Moreover, the Gauge invariant quantity $u=\log |\phi|^{2}$ satisfies

$$
-\Delta u=F_{12}-4 \pi \sum_{j=1}^{N} \alpha_{j} \delta_{p_{j}} .
$$

Putting together (3) with (2) we arrive at the following elliptic equation:

$$
-\Delta u=\frac{4}{k^{2}} e^{u}\left(1-e^{u}\right)-4 \pi \sum_{j=1}^{N} \alpha_{j} \delta_{p_{j}} .
$$

Conversely, given $u$ be a solution of (4) we can recast a vortex solution $(\mathcal{A}, \phi)$ for the selfdual system by setting

$$
\left\{\begin{array}{l}
\phi=e^{\frac{u}{2}+i \sum_{j=1}^{N} \alpha_{j} \operatorname{Arg}\left(z-p_{j}\right)} \\
A_{1}+i A_{2}=-i \partial_{+} \log \phi \\
A_{0}=\frac{1}{k}\left(1-|\phi|^{2}\right)
\end{array}\right.
$$

This point of view has been used for the first time by Taubes for the abelian Maxwell-Higgs theory [Ta] and it reduces the search of selfdual Chern-Simons vortices to solve some semilinear elliptic equation.

\section{A "model" problem}

Motivated by equations in the form of (4), we are interested to analyze the elliptic equation:

$$
-\Delta u=V(z) e^{u}-4 \pi \sum_{j=1}^{N} \alpha_{j} \delta_{p_{j}} \text { in } \Omega,
$$


where $\Omega$ is a smooth domain in $\mathbb{R}^{2}, p_{1}, \ldots, p_{N}$ are assigned distinct points in $\Omega, \alpha_{i}>0$ and $0<a \leq$ $V(z) \leq b$. In terms of the regular part $v$ of $u$, we are lead equivalently to study:

$$
-\Delta v=\left(\prod_{j=1}^{N}\left|z-p_{j}\right|^{2 \alpha_{j}}\right) V(z) e^{v} \text { in } \Omega .
$$

Equations of the form:

$$
-\Delta v=|z|^{2 \alpha} V(z) e^{v} \text { in } \Omega, \alpha \geq 0,0 \in \Omega,
$$

supplemented by some finite energy condition, are "almost"invariant under the scale transformation:

$$
v(z) \rightarrow v_{\mu}(z)=v\left(\frac{z}{\mu}\right)-2(\alpha+1) \log \mu, \mu>0,
$$

which is responsible for a compactness failure and it relates to solutions for the limit problem:

$$
\left\{\begin{array}{l}
-\Delta v=|z|^{2 \alpha} e^{v} \text { in } \mathbb{R}^{2} \\
\int_{\mathbb{R}^{2}}|z|^{2 \alpha} e^{v}<+\infty
\end{array}\right.
$$

By the results in $[\mathrm{CLi}],[\mathrm{CW}],[\mathrm{Lio}]$ and $[\mathrm{PT}]$, a complete classification is available and gives that

$$
v(z)=\log \frac{8 \mu^{2}(\alpha+1)^{2}}{\left(\mu^{2}+\left|z^{\alpha+1}-c\right|^{2}\right)^{2}}, \mu>0, c \in \mathbb{C},
$$

with $c=0$ if $\alpha \notin \mathbb{N}$. Note that

$$
\int_{\mathbb{R}^{2}}|z|^{2 \alpha} e^{v}=8 \pi(\alpha+1) .
$$

There are a lot of deep results on this topic, from a Concentration/Compactness Principle to a complete description of the blow up profile, from sup + inf-inequalities of Harnack type to a general degree formula for the associated Fredholm map. We refer to [Ta3] for an account on the subject which show that, while the situation is completely understood for $\alpha=0$, it presents still intriguing aspects in case $\alpha>0$.

We address now the question of constructing blow up solutions for the "model" problem since, for instance, in vortex theory the interest in "blowing up" solutions is related to the presence of vortices with strongly localized electromagnetic field.

For this purpose, we need to impose appropriate boundary conditions, and we consider Dirichlet boundary conditions even though for the physical applications other choices could be relevant, such as the periodic one.

Let us consider a sequence $v_{n}$ satisfying:

$$
\begin{cases}-\Delta v_{n}=\rho_{n}^{2} K(z) e^{v_{n}} & \text { in } \Omega \\ v_{n}=0 & \text { on } \partial \Omega\end{cases}
$$

with $K(z)=\left(\prod_{j=1}^{N}\left|z-p_{j}\right|^{2 \alpha_{j}}\right) V(z), \rho_{n} \rightarrow \rho \geq 0$ and $\lambda_{n}=\rho_{n}^{2} \int_{\Omega} K(z) e^{v_{n}} \rightarrow \lambda$.

Denote by $G\left(z, z^{\prime}\right)$ the Green's function of $-\Delta$ with Dirichlet boundary condition on $\Omega$ and $H\left(z, z^{\prime}\right)$ its regular part defined as $H\left(z, z^{\prime}\right)=G\left(z, z^{\prime}\right)+\frac{1}{2 \pi} \log \left|z-z^{\prime}\right|$. Set $\Gamma=\left\{p_{1}, . ., p_{N}\right\}$ and $\Omega^{\prime}=\Omega \cap\{K>0\}$, and for given $m \in \mathbb{N}$ and $s \in\{1, . ., N\}$ define

$$
\tilde{\mathcal{F}}\left(z_{1}, . ., z_{m}\right)=\sum_{i=1}^{m} H\left(z_{i}, z_{i}\right)+\sum_{i \neq j} G\left(z_{i}, z_{j}\right)+\frac{1}{4 \pi} \sum_{i=1}^{m} \ln K\left(z_{i}\right)
$$


which is well defined in $\left(\Omega^{\prime}\right)^{m}$ for $z_{i} \neq z_{j}$ for $i \neq j$, and let

$$
\mathcal{G}\left(z_{1}, . ., z_{m}, \omega_{1}, . ., \omega_{s}\right)=\frac{1}{4 \pi}\left(\sum_{i=1}^{m} \sum_{j=1}^{s} 8 \pi\left(1+\alpha_{j}\right) G\left(z_{i}, \omega_{j}\right)\right)
$$

well defined for $z_{i} \neq \omega_{j}$, with $z_{i} \in \Omega, \omega_{j} \in \mathbb{C}, i=1, . ., m, j=1, . ., s$. An extension to the singular case of the blow up analysis in [BM] is due to [BT2] (see also [BT1]) and permits to perform an asymptotic analysis in the spirit of $[\mathrm{MW}]$ and $[\mathrm{Su}]$ for the regular case:

Theorem 1. [Es2] Let $V$ be a smooth positive function in $\bar{\Omega}$.

a) If either $\rho>0$ or $\lambda<8 \pi$, then (up to a subsequence) $v_{n} \rightarrow v_{0}$ in $C^{2, \beta}(\Omega)$, and $v_{0}$ satisfies:

$$
\begin{cases}-\Delta v_{0}=\rho^{2}\left(\prod_{i=1}^{N}\left|z-p_{i}\right|^{2 \alpha_{i}}\right) V e^{v_{0}} & \text { in } \Omega \\ v_{0}=0 & \text { on } \partial \Omega,\end{cases}
$$

with $\beta=\frac{1}{2} \min \left\{\alpha_{1}, \ldots, \alpha_{N}\right\}$. In particular, for $\lambda=0 v_{n} \rightarrow 0$ in $C^{2, \beta}(\Omega)$ and, for $n$ large, $v_{n}$ coincides with the unique minimal solution of (5) (see $[C R])$.

b) If $\rho_{n} \rightarrow 0$ and $\lambda_{n} \rightarrow \lambda \geq 8 \pi$, then (up to a subsequence) there exists a non empty finite set $S=\left\{q_{1}, . ., q_{K}\right\} \subset \Omega$ (blow up set) such that

$$
\rho_{n}^{2} K(z) e^{v_{n}}-\sum_{i=1}^{K} b_{i} \delta_{q_{i}} \text { in the sense of measures }
$$

and $v_{n} \rightarrow \sum_{i=1}^{K} b_{i} G\left(z, q_{i}\right)$ in $C_{l o c}^{2, \beta}(\bar{\Omega} \backslash S)$, with $b_{i}=8 \pi$ if $q_{i} \notin \Gamma$, or $b_{i}=8 \pi\left(1+\alpha_{j}\right)$ if $q_{i}=p_{j}$ for some $j=1, . ., N$. Moreover:

if $S \cap \Gamma=\emptyset$, then $\left(q_{1}, . ., q_{K}\right)$ is a critical point for the function $\tilde{\mathcal{F}}$;

if $S \cap \Gamma=\left\{p_{j_{1}}, . ., p_{j_{s}}\right\}$ and $S \backslash \Gamma=\left\{q_{i_{1}}, . ., q_{i_{m}}\right\}$, then $\left(q_{i_{1}}, . ., q_{i_{m}}\right)$ is a critical point for the function $\tilde{\mathcal{F}}+\mathcal{G}\left(\cdot, p_{j_{1}}, . ., p_{j_{s}}\right)$.

In the sequel, according to whether $S \cap \Gamma=\emptyset$ or not, we will denote by $\mathcal{F}$ the function $\tilde{\mathcal{F}}$ or $\tilde{\mathcal{F}}+\mathcal{G}$, and we will speak of concentration whenever condition (6) holds.

We address now the existence of blowing up solutions of (5) for a fixed sequence $\rho_{n} \rightarrow 0$. Physical considerations suggest that concentration is more likely to occur at the vortex points $p_{1}, \ldots, p_{N}$ as the following result shows in a rigorous way:

Theorem 2. [Es1] Fix $\left\{p_{j_{1}}, \ldots, p_{j_{s}}\right\} \in \Omega \cap\{V>0\}$ and assume $\alpha_{j_{i}} \notin \mathbb{N}$. For $n$ large, there exists a solution sequence $v_{n}$ of (5) concentrating in $\left\{p_{j_{1}}, \ldots, p_{j_{s}}\right\}$ as $n \rightarrow+\infty$.

According to Theorem 1, we know that concentration outside the vortex points is possible only for particular configurations of blow up points. We have:

Theorem 3. [Es1] Fix $\left\{p_{j_{1}}, \ldots, p_{j_{s}}\right\}$ and let $\left\{q_{1}, \ldots, q_{k}\right\}$ be a non degenerate critical point of $\mathcal{F}$ such that $\Delta \log V\left(q_{i}\right)=0$. For $n$ large, there exists a solution sequence $v_{n}$ of (5) concentrating in $\left\{p_{j_{1}}, \ldots, p_{j_{s}}, q_{1}, \ldots, q_{k}\right\}$ as $n \rightarrow+\infty$, provided $\alpha_{j_{i}} \notin \mathbb{N}$.

Both Theorem 2 and Theorem 3 have been inspired by an approach developed by Baraket and Pacard for the regular problem, i.e. $\left\{p_{1}, \ldots, p_{N}\right\}=\emptyset$, in $[\mathrm{BP}]$. In fact, if no singular sources are present, Theorem 3 gives a direct extension of Baraket-Pacard's result to a non constant weight function $V(z)$.

In addition, for the regular problem, in collaboration with M. Grossi and A. Pistoia, we have introduced in [EGP] a different approach in order to remove the assumption on $\Delta \log V$ and to replace 
the non degeneracy condition for the critical point $\left(q_{1}, \ldots, q_{k}\right)$ of $\mathcal{F}$ with a milder stability notion, which holds in particular for strict local minima or maxima and for isolated critical points with non zero topological index. The approach can be used also for the singular problem in the "not-integer" case and gives the general result:

Theorem 4. Fix $\left\{p_{j_{1}}, \ldots, p_{j_{s}}\right\}$ and let $\left\{q_{1}, \ldots, q_{k}\right\}$ be a "stable" critical point of $\mathcal{F}$. For n large, there exists a solution sequence $v_{n}$ of (5) concentrating in $\left\{p_{j_{1}}, \ldots, p_{j_{s}}, q_{1}, \ldots, q_{k}\right\}$ as $n \rightarrow+\infty$, provided $\alpha_{j_{i}} \notin \mathbb{N}$.

For the proof of Theorem 4 in case $\left\{p_{j_{1}}, \ldots, p_{j_{s}}\right\}=\emptyset$ we refer to [EGP]. We point out that this method could be very promising in order to handle the singular problem when the Dirac measures appear with integral multiplicities, as needed in the physical applications.

\section{Proof of Theorem 1}

The first ingredient is an asymptotic result due to Brezis-Merle $[\mathrm{BM}]$ for the regular case and extended by Bartolucci-Tarantello [BT2] to the singular case (see also [BCLT], [BT1], [Ta1]-[Ta2] for some insight on the singular case):

Theorem 5. [BT2] Let $v_{n}$ be a solution sequence of

$$
\left\{\begin{array}{l}
-\Delta v_{n}=|z|^{2 \alpha_{n}} V_{n}(z) e^{v_{n}} \\
\int_{\Omega}|z|^{2 \alpha_{n}} V_{n}(z) e^{v_{n}} \leq C .
\end{array}\right.
$$

Assume $\alpha_{n} \rightarrow \alpha \geq 0,0<a \leq V_{n} \leq b$ and $\left|\nabla V_{n}\right| \leq A$ in $\Omega$. There exists a subsequence $\left\{v_{n_{k}}\right\}$ satisfying one of the following alternatives:

either

i) $\left\{v_{n_{k}}\right\}$ is bounded in $L_{\text {loc }}^{\infty}(\Omega)$

or

ii) $v_{n_{k}} \rightarrow-\infty$ uniformly on compact subsets of $\Omega$

or

iii) the blow up set $S=\cup_{i}\left\{q_{i}\right\}$ relative to $\left\{v_{n_{k}}\right\}$ is finite and non empty, $v_{n_{k}} \rightarrow-\infty$ uniformly on compact subsets of $\Omega \backslash S$ and $|z|^{2 \alpha_{n_{k}}} V_{n_{k}} e^{v_{n_{k}}} \rightarrow \sum_{i} b_{i} \delta_{q_{i}}$ weakly in the sense of measures on $\Omega$, where $b_{i} \geq 8 \pi$.

The second ingredient is contained in following Pohozaev-type identities:

Lemma 6. [Po] For any solution $v \in C^{2}(\Omega) \cap C^{1}(\bar{\Omega})$ of $-\Delta v=K e^{v}$ in $\Omega$ we have:

$$
\begin{gathered}
\int_{\Omega}\left(2 K+<\nabla K, z-z_{0}>\right) e^{v} \quad \int_{\partial \Omega}<z-z_{0}, \nu>K e^{v}+\int_{\partial \Omega}<z-z_{0}, \nabla v>\frac{\partial v}{\partial \nu} \\
-\frac{1}{2} \int_{\partial \Omega}<z-z_{0}, \nu>|\nabla v|^{2}, \\
\int_{\Omega} \frac{\partial K}{\partial z_{i}} e^{v}=\int_{\partial \Omega} K e^{v} \nu_{i}+\int_{\partial \Omega} \frac{\partial v}{\partial z_{i}} \frac{\partial v}{\partial \nu}-\frac{1}{2} \int_{\partial \Omega}|\nabla v|^{2} \nu_{i}
\end{gathered}
$$

for $i=1,2$ and $z_{0} \in \mathbb{R}^{2}$, where $\nu(z)$ is the unit outer normal vector of $\partial \Omega$ at $z \in \partial \Omega$.

The third ingredient is an $L^{\infty}$-bound near the boundary for solutions with homogeneous Dirichlet boundary condition, which is a special feature of dimension two. We have the following result: 
Lemma 7. [MW] Let $K$ be a function in $\bar{\Omega}$ such that $K>0$ and $|\nabla \log K| \leq A$ in a closed neighbourhood $U$ of $\partial \Omega$. There exists $d>0$, depending only on $A$ and $\Omega$, such that if $v$ satisfies: $-\Delta v=K e^{v}$ in $\Omega, v=0$ on $\partial \Omega$, then

$$
\max _{\{z \in \Omega: \operatorname{dist}(z, \partial \Omega) \leq d\}} v \leq \max _{\{z \in \Omega: \operatorname{dist}(z, \partial \Omega)=d\}} v .
$$

Proof Let $z_{0} \in \partial \Omega$, and $r>0$ sufficiently small such that $\overline{B\left(z_{0}+r \nu_{\Omega}\left(z_{0}\right), r\right)} \cap \bar{\Omega}=\left\{z_{0}\right\}$, where $\nu_{\Omega}(z)$ denotes the unit outer normal vector of $\partial \Omega$ at $z \in \partial \Omega$.

Set $z_{1}=z_{0}+r \nu_{\Omega}\left(z_{0}\right)$, and define the Kelvin tranform of $v$ as given by $\hat{v}(y)=v\left(z_{1}+r^{2} \frac{y-z_{1}}{\left|y-z_{1}\right|^{2}}\right)$ for $y \in$ $\hat{\Omega}=\left\{y=z_{1}+r^{2} \frac{z-z_{1}}{\left|z-z_{1}\right|^{2}}: z \in \Omega\right\}$. We have: $-\Delta \hat{v}=\hat{K} e^{\hat{v}}$ in $\hat{\Omega}$, with $\hat{K}(y)=\frac{r^{4}}{\left|y-z_{1}\right|^{4}} K\left(z_{1}+r^{2} \frac{y-z_{1}}{\left|y-z_{1}\right|^{2}}\right)$. We easily compute,

$$
\frac{\partial \log \hat{K}}{\partial y_{i}}(y)=-4 \frac{\left(y-z_{1}\right)_{i}}{\left|y-z_{1}\right|^{2}}+\frac{\partial \log K}{\partial z_{j}}\left(z_{1}+r^{2} \frac{y-z_{1}}{\left|y-z_{1}\right|^{2}}\right)\left(\frac{r^{2}}{\left|y-z_{1}\right|^{2}} \delta_{i j}-2 r^{2} \frac{\left(y-z_{1}\right)_{i}\left(y-z_{1}\right)_{j}}{\left|y-z_{1}\right|^{4}}\right),
$$

and observe that $\nu_{\hat{\Omega}}\left(z_{0}\right)=-\nu_{\Omega}\left(z_{0}\right)$. Since $K>0$, we derive $<\nabla \hat{K}(y), \nu_{\hat{\Omega}}\left(z_{0}\right)><0$ for $y \in \hat{\Omega}$ close to $z_{0}$ and $r>0$ sufficiently small (depending on $z_{0}, A$ and $\Omega$ ). At this point, to analyze $\hat{v}$ we can use the moving plane technique as developed in [GNN]. In fact, $\hat{\Omega}$ is strictly convex in $z_{0}$ and $\hat{K}$ decreases near $z_{0}$ along the direction $\nu_{\hat{\Omega}}\left(z_{0}\right)$. Moving along a plane orthogonal to $\nu_{\hat{\Omega}}\left(z_{0}\right)$, we can prove that in a neighbourhood of $z_{0}$ in $\hat{\Omega}$ (depending only on $z_{0}, A$ and $\Omega$ ) the function $\hat{v}$ has no critical points.

This reflects on $v$ ensuring that there exists a neighbourhood $U_{z_{0}}$ of $z_{0}$ in $\Omega$ such that $v$ has no critical points in $U_{z_{0}}$. Moving $z_{0}$ in the compact set $\partial \Omega$, we can find $d>0$, depending only on $A$ and $\Omega$, such that $v$ has no critical points in $\{z \in \Omega$ : dist $(z, \partial \Omega) \leq d\}$ and then (10) holds.

We are now ready for:

Proof (of Theorem 1) a) First of all notice that, in view of Lemma 7 there exists $\Omega^{\prime} \subset \subset \Omega$ relatively compact in $\Omega$ such that the set of blow up points of any subsequence of $v_{n}$ is contained in $\Omega^{\prime}$ and

$$
\sup _{\Omega^{\prime}} v_{n}=\sup _{\Omega} v_{n} .
$$

We apply Theorem 5 to $\xi_{n}=v_{n}+2 \log \rho_{n}$. If $\lambda<8 \pi$, then we can exclude alternative (iii) along any subsequence, and obtain that $\xi_{n}$ is locally uniformly bounded from above in $\Omega$. If $\rho>0$, the positivity of $v_{n}$ implies that $\xi_{n}$ is uniformly bounded from below in $\bar{\Omega}$ and again, we can exclude alternatives (ii) and (iii) along any subsequence, and conclude that $\xi_{n}$ is locally uniformly bounded in $\Omega$.

Hence, thanks to (11), in any case we conclude that $\xi_{n}$ is uniformly bounded from above in $\bar{\Omega}$, and by the relation $\rho_{n}^{2} K e^{v_{n}}=K e^{\xi_{n}}$, we deduce that $\rho_{n}^{2} K e^{v_{n}}$ is uniformly bounded in $\bar{\Omega}$. By elliptic regularity theory (see $[\mathrm{GT}]$ ) we get that $v_{n}$ is bounded in $C^{1, \alpha}(\Omega)$ for any $\alpha \in(0,1)$, and we can take a subsequence with the desired convergence property.

b) Apply Theorem 5 to $\xi_{n}$ and note that alternatives (i) and (ii) cannot hold. Otherwise, as in the previous part a), (up to a subsequence) $\xi_{n}$ would be uniformly bounded from above in $\bar{\Omega}$, and since $-\Delta v_{n}=\rho_{n}^{2} K e^{v_{n}}=K e^{\xi_{n}}, v_{n}=0$ on $\partial \Omega$, we would also get that $v_{n}$ is uniformly bounded in $\bar{\Omega}$. Consequently, $\lambda_{n}=\rho_{n}^{2} \int_{\Omega} K e^{v_{n}} \rightarrow 0$, as $n \rightarrow+\infty$, in contradiction with the fact that $\lambda_{n} \rightarrow \lambda>0$. So, alternative (iii) must hold and there exists a finite set $S=\cup_{i}\left\{s_{i}\right\}$ (blow up set) such that (up to a subsequence) $\xi_{n} \rightarrow-\infty$ uniformly on compact sets in $\Omega \backslash S$ and $K e^{\xi_{n}}=\rho_{n}^{2} K e^{v_{n}} \rightarrow \sum b_{i} \delta_{s_{i}}$ weakly in the sense of measures with $b_{i} \geq 8 \pi$. In particular, by Lemma $7 \rho_{n}^{2} K e^{v_{n}}=K e^{\xi_{n}} \rightarrow 0$ uniformly on compact sets in $\bar{\Omega} \backslash S$. 
Let $\varepsilon>0$ small. By Green's representation formula, we get that

$$
\begin{aligned}
\left|v_{n}(z)-\sum_{i} b_{i} G\left(z, s_{i}\right)\right| & =\left|\rho_{n}^{2} \int_{\Omega} G(z, y) K(y) e^{v_{n}(y)} d y-\sum_{i} b_{i} G\left(z, s_{i}\right)\right| \\
& \leq\left|\rho_{n}^{2} \sum_{i} \int_{B\left(s_{i}, \varepsilon\right)} G(z, y) K(y) e^{v_{n}(y)} d y-\sum_{i} b_{i} G\left(z, s_{i}\right)\right|+o_{n}(1) \\
& \leq\left|\rho_{n}^{2} \sum_{i} \int_{B\left(s_{i}, \varepsilon\right)}\left(G(z, y)-G\left(z, s_{i}\right)\right) K(y) e^{v_{n}(y)} d y\right|+o_{n}(1) \\
& \leq C \sum_{i} \sup _{\left|y-s_{i}\right| \leq \varepsilon}\left|G(z, y)-G\left(z, s_{i}\right)\right|+o_{n}(1) \rightarrow 0
\end{aligned}
$$

as $n \rightarrow+\infty$ and $\varepsilon \rightarrow 0$, uniformly on the set $\Omega_{\delta}:=\{z \in \Omega: \operatorname{dist}(z, S) \geq \delta\}$ for fixed small $\delta>0$. We have used the property that $\sum_{i} \sup _{\left|y-s_{i}\right| \leq \varepsilon}\left|G(z, y)-G\left(z, s_{i}\right)\right| \rightarrow 0$ as $\varepsilon \rightarrow 0$ uniformly on $\Omega_{\delta}$. Similarly, we can handle the derivatives of $v_{n}$ and prove that $v_{n} \rightarrow \sum_{i} b_{i} G\left(z, s_{i}\right)$ uniformly in $C_{\text {loc }}^{1}(\bar{\Omega} \backslash S)$. Since $-\Delta\left(v_{n}-\sum_{i} b_{i} G\left(z, s_{i}\right)\right)=\rho_{n}^{2} K e^{v_{n}}$ in $\Omega_{\delta}$, with $\rho_{n}^{2} K e^{v_{n}} \rightarrow 0$ in $C^{0, \beta}\left(\Omega_{\delta}\right)$ and $\max _{\partial \Omega_{\delta}} \mid v_{n}-$ $\sum_{i} b_{i} G\left(z, s_{i}\right) \mid \rightarrow 0$, we can use elliptic regularity to conclude that, in fact,

$$
v_{n} \rightarrow \sum_{i} b_{i} G\left(z, s_{i}\right) \text { in } C_{\mathrm{loc}}^{2, \beta}(\bar{\Omega} \backslash S) .
$$

Now we use (8) on $B_{i}=B_{\varepsilon}\left(s_{i}\right)$ with $z_{0}=s_{i}$ and $\varepsilon$ sufficiently small and we obtain

$$
\rho_{n}^{2} \int_{B_{i}}\left(2 K+<\nabla K, z-s_{i}>\right) e^{v_{n}}=\varepsilon \rho_{n}^{2} \int_{\partial B_{i}} K e^{v_{n}}+\varepsilon \int_{\partial B_{i}}\left(\frac{\partial v_{n}}{\partial \nu}\right)^{2}-\frac{\varepsilon}{2} \int_{\partial B_{i}}\left|\nabla v_{n}\right|^{2} .
$$

For $\varepsilon$ small, for the left hand side we have:

$$
\begin{aligned}
\text { L.H.S. } & =2 b_{i}+o_{n}(1)+\rho_{n}^{2} \int_{B_{i}}\left(\prod_{j=1}^{N}\left|z-p_{j}\right|^{2 \alpha_{j}}\right)<\nabla V, z-s_{i}>e^{v_{n}} \\
& +\rho_{n}^{2} \int_{B_{i}}<2 \sum_{j=1}^{N} \alpha_{j} \frac{z-p_{j}}{\left|z-p_{j}\right|^{2}}, z-s_{i}>K e^{v_{n}} \rightarrow\left\{\begin{array}{l}
2 b_{i} \text { if } s_{i} \notin \Gamma \\
2\left(1+\alpha_{j}\right) b_{i} \text { if } s_{i}=p_{j} \in \Gamma
\end{array}\right.
\end{aligned}
$$

While, for the right hand side we have:

$$
\begin{aligned}
\text { R.H.S. } & \rightarrow \varepsilon \int_{\partial B_{i}}\left(\sum_{j} b_{j} \frac{\partial G}{\partial \nu}\left(z, s_{j}\right)\right)^{2}-\frac{\varepsilon}{2} \int_{\partial B_{i}}\left|\sum_{j} b_{j} \nabla G\left(z, s_{j}\right)\right|^{2} \\
& =\varepsilon\left[\int_{\partial B_{i}} b_{i}^{2}\left(\frac{\partial G}{\partial \nu}\left(z, s_{i}\right)\right)^{2}-\frac{1}{2} \int_{\partial B_{i}} b_{i}^{2}\left|\nabla G\left(z, s_{i}\right)\right|^{2}+O(1)\right]=\frac{b_{i}^{2}}{4 \pi}+O(\varepsilon) .
\end{aligned}
$$

Passing to the limit as $\varepsilon \rightarrow 0$ we find that $b_{i}=8 \pi$ if $s_{i} \notin \Gamma$ and $b_{i}=8 \pi\left(\alpha_{j}+1\right)$ if $s_{i}=p_{j} \in \Gamma$.

Now, let $S \backslash \Gamma=\left\{q_{1}, \ldots, q_{k}\right\}$, and use (9) on $B_{i}=B_{\varepsilon}\left(q_{i}\right)$ with $z_{0}=q_{i}$ and $\varepsilon$ sufficiently small to obtain:

$$
\rho_{n}^{2} \int_{B_{i}} \frac{\partial K}{\partial z_{k}} e^{v_{n}}=\rho_{n}^{2} \int_{\partial B_{i}} K e^{v_{n}} \nu_{k}+\int_{\partial B_{i}} \frac{\partial v_{n}}{\partial z_{k}} \frac{\partial v_{n}}{\partial \nu}-\frac{1}{2} \int_{\partial B_{i}}\left|\nabla v_{n}\right|^{2} \nu_{k}, \quad k=1,2 .
$$

For the left hand side we get:

$$
\text { L.H.S. }=\rho_{n}^{2} \int_{B_{i}} \frac{\partial}{\partial z_{k}}(\log K) K e^{v_{n}} \rightarrow 8 \pi \frac{\partial}{\partial z_{k}}(\log K)\left(q_{i}\right), \quad \text { as } n \rightarrow+\infty .
$$


Setting $\mathcal{F}_{i}(z)=8 \pi H\left(z, q_{i}\right)+\sum_{j \neq i} b_{j} G\left(z, q_{j}\right)$ for $z \neq q_{j}, j \neq i$, for the right hand side we have:

$$
\begin{aligned}
\text { R.H.S. } & \rightarrow \int_{\partial B_{i}}\left(-4 \frac{\left(z-q_{i}\right)_{k}}{\left|z-q_{i}\right|^{2}}+\frac{\partial \mathcal{F}_{i}}{\partial z_{k}}\right)\left(-\frac{4}{\varepsilon}+\frac{\partial \mathcal{F}_{i}}{\partial \nu}\right)-\frac{1}{2} \int_{\partial B_{i}}\left|-4 \frac{z-q_{i}}{\left|z-q_{i}\right|^{2}}+\nabla \mathcal{F}_{i}\right|^{2} \frac{\left(z-q_{i}\right)_{k}}{\left|z-q_{i}\right|} \\
& =-\frac{4}{\varepsilon} \int_{\partial B_{i}} \frac{\partial \mathcal{F}_{i}}{\partial z_{k}}+O(\varepsilon) .
\end{aligned}
$$

Passing to the limit as $\varepsilon \rightarrow 0$ we find that

$$
8 \pi \nabla\left(\log K+\mathcal{F}_{i}\right)\left(q_{i}\right)=0
$$

as claimed.

\section{$5 \quad$ Existence of blowing up solutions}

In order to describe the idea beyond Theorems 2-4, we will restrict to the simple case where the concentration set $S$ reduces to a single point. Our construction relies in an essential way:

- on the choice of a suitable family of approximating solutions $V_{n}$;

- on the invertibility, in some sense, of the linearized operator at such approximating solution.

Since we are describing a "single" peak blow up, we denote it by

- $p$ if it belongs to $\left\{p_{1}, \ldots, p_{N}\right\}$ and $\alpha$ is the corresponding multiplicity (cfr. Theorem $2 / 4$ );

- $q$ if it does not belong to $\left\{p_{1}, \ldots, p_{N}\right\}$ and is a critical point of $\mathcal{F}(z)$ (cfr. Theorem 3/4).

Moreover, let us represent the weight function $K(z)$ in (5) in the form $K(z)=|z-p|^{2 \alpha} V(z)$.

Let us concentrate first in Theorems 2-3. Following Baraket and Pacard, the aim is to construct a "local profile" around $p$ (or $q$ ) which is "glued" with the Green's profile $L(z)=8 \pi(1+\alpha) G(z, p)$ (or $L(z)=8 \pi G(z, q)$ ) away from it. Inspired by the classification result for the limit problem, we can define

$$
v_{\tau}(z)=\log \frac{8 \tau^{2}}{\left(\tau^{2} \rho_{n}^{2}+|z|^{2(\alpha+1)}\right)^{2}}
$$

which is a solution for

$$
-\Delta v_{\tau}=\rho_{n}^{2}|z|^{2 \alpha} e^{v_{\tau}} \text { in } \mathbb{R}^{2}
$$

We work in some weighted Hölder-type spaces (introduced for the first time in [CHS]), appropriate in order to have a good control for the invertibility of the linearized operator, and we need to take an approximating function sufficiently accurate with respect to these norms. Since $\rho_{n} \rightarrow 0$, the function $v_{\tau}$ peaks in the origin and it is reasonable to construct an approximating function $V_{n}$ which looks like $v_{\tau}(z-p)-\log V(p)$ in $p$, for a suitable choice of $\tau$. The construction is accurate in $p$. As far as $q$, first we consider a local peak $v_{\tau, \gamma}(z-q), \gamma \in \mathbb{C}$, where

$$
v_{\tau, \gamma}(z)=\log \frac{8 \tau^{2}\left|1+3 \gamma z^{2}\right|^{2}}{\left(\tau^{2} \rho_{n}^{2}+|z|^{2}\left|1+\gamma z^{2}\right|^{2}\right)^{2}}
$$

is a solution for

$$
-\Delta v_{\tau, \gamma}=\rho_{n}^{2} e^{v_{\tau, \gamma}} \text { in }\left\{1+3 \gamma z^{2} \neq 0\right\}
$$

and, for a suitable choice of $\gamma$, we are able to reduce the invertibility of the linearized operator to the invertibility of the Hessian of $\mathcal{F}$ at $q$. Secondly, we need to be more accurate in $q$, in view of the more degeneracy due to the translations, by taking a local peak of the form $v_{\tau, \gamma}(z-q)-P_{2}(z)$, where $P_{2}(z)$ is the second-order Taylor polinome of $\log K(z)$ in $q$. By construction, near $p$ we have that

$$
\Delta V_{n}+\rho_{n}^{2}|z-p|^{2 \alpha} V(z) e^{V_{n}}=O\left(\rho_{n}^{2}|z-p|^{2 \alpha+1} e^{V_{n}}\right)
$$


and near $q$ we have that

$$
\Delta V_{n}+\rho_{n}^{2} K(z) e^{V_{n}}=O\left(\rho_{n}^{2}|z-q|^{3} e^{V_{n}}\right)
$$

provided $\Delta \log V(q)=0$. Now we have to control what it happens in the intermediate zone. The following expansion holds as $n \rightarrow+\infty$ :

$$
V_{n}(z)-L(z) \sim \ln \left(8(1+\alpha)^{2} \tau^{2}\right)-\mathcal{F}_{1}(p)+O(|z-p|)=O(|z-p|) \text { as } z \sim p
$$

if we choose $\tau=\frac{e^{\frac{1}{2} \mathcal{F}_{1}(p)}}{\sqrt{8}(1+\alpha)}$, where $\mathcal{F}_{1}(z)=8 \pi(1+\alpha) H(z, p)+\ln V(z)$;

$V_{n}(z)-L(z) \sim \ln \left(8 \tau^{2}\right)-\mathcal{F}_{2}(q)-\operatorname{Re}\left[\left(\partial_{z z} \mathcal{F}_{2}(q)-2 \gamma\right)(z-q)^{2}\right]+O\left(|z-q|^{3}\right)=O\left(|z-q|^{3}\right)$ as $z \sim q$

if we choose $\tau=\frac{e^{\frac{1}{2} \mathcal{F}_{2}(q)}}{\sqrt{8}}$ and $\gamma=\frac{1}{2} \partial_{z z} \mathcal{F}_{2}(q)$, where $\mathcal{F}_{2}(z)=8 \pi H(z, q)+\ln K(z)$. Let us remark that the criticality assumption for $q$ reads in this context as $\partial_{z} \mathcal{F}_{2}(q)=0$.

The accuracy now is satisfactory and, in view of the invertibility (in some sense) of the linearized operator, it is possible to conclude with the aid of a fixed point argument.

We describe now an alternative approach to the existence of "concentrating" solutions, in the spirit of some perturbation method available in higher dimension (cfr. [Ba], [Re]). We introduce a perturbation setting in the space $H_{0}^{1}(\Omega)$ and try to replace the non-degeneracy assumption for the critical point of $\mathcal{F}$ (often very difficult to check in the applications) with a weaker "stable" condition.

We define

$$
v_{\tau, \theta}(z)=v_{\tau}(z-\theta)=\log \frac{8 \tau^{2}}{\left(\tau^{2} \rho_{n}^{2}+|z-\theta|^{2(\beta+1)}\right)^{2}}
$$

with $\beta=\alpha$ if $S=\{p\}$ and $\beta=0$ if $S=\{q\}$. We look for solutions close in $H_{0}^{1}(\Omega)$ to $P v_{\tau, p}$ for a suitable $\tau>0$ in case $S=\{p\}$. While, we look for solutions of the form $P v_{\tau_{n}, \xi_{n}}+\phi_{n}$, for suitable $\tau_{n}>0, \xi_{n}$ close to $q$ and $\phi_{n}$ a small function in a suitable space, in case $S=\{q\}$. Here, $P v_{\tau, \theta}$ denotes the projection of $v_{\tau, \theta}$ into $\mathrm{H}_{0}^{1}(\Omega)$, in other words, $P v_{\tau, \theta}$ is uniquely defined as satisfying:

$$
\begin{cases}-\Delta P v_{\tau, \theta}=-\Delta v_{\tau, \theta}=\rho_{n}^{2}|z-\theta|^{2 \beta} e^{v_{\tau, \theta}} & \text { in } \Omega \\ P v_{\tau, \theta}=0 & \text { on } \partial \Omega\end{cases}
$$

Since

$$
P v_{\tau, \theta}(z)-v_{\tau, \theta}-8 \pi(\beta+1) H(\cdot, \theta)+\left.\log \left(8 \tau^{2}\right)\right|_{\partial \Omega}=O\left(\rho_{n}^{2}\right),
$$

by harmonicity we have

$$
P v_{\tau, \theta}(z)=v_{\tau, \theta}+8 \pi(\beta+1) H(\cdot, \theta)-\log \left(8 \tau^{2}\right)+O\left(\rho_{n}^{2}\right)
$$

in $C^{0}(\bar{\Omega})$ uniformly for $\theta$ away from $\partial \Omega$. Hence, the function $P v_{\tau, \theta}$ "almost" satisfies:

$$
-\Delta P v_{\tau, \theta}=\rho_{n}^{2}|z-\theta|^{2 \beta} e^{v_{\tau, \theta}} \sim 8 \tau^{2} \rho_{n}^{2}|z-\theta|^{2 \beta} e^{-8 \pi(\beta+1) H(z, \theta)} e^{P v_{\tau, \theta}} .
$$

In case $S=\{p\}$, we choose $\tau(p)=\sqrt{\frac{V(p)}{8}} e^{4 \pi(\beta+1) H(p, p)}$, and in case $S=\{q\}$, we choose $\tau(\xi)=$ $\sqrt{\frac{K(\xi)}{8}} e^{4 \pi H(\xi, \xi)}$ for any $\xi \in \Omega^{\prime}=\Omega \cap\{K>0\}$. Thanks to this choice, we obtain that $P v_{\tau(p), p}$ and $P v_{\tau(\xi), \xi}$ are approximating solutions. Define the operator $L$ in the form:

$$
L=\Delta+\rho_{n}^{2}|z-p|^{2 \alpha} e^{v_{\tau, p}} \text { if } S=\{p\} \quad \text { or } \quad L=\Delta+\rho_{n}^{2} e^{v_{\tau, \xi}} \text { if } S=\{q\}, \xi \in \Omega^{\prime},
$$

and set

$$
\psi_{\tau, \theta}^{0}=\frac{\partial v_{\tau, \theta}}{\partial \tau}, \psi_{\tau, \theta}^{j}=\frac{\partial v_{\tau, \theta}}{\partial \theta_{j}}, j=1,2 .
$$


In a suitable functional space, we have that

$$
\text { ker } L=\operatorname{Span}\left\{\psi_{\tau, p}^{0}\right\} \text { if } S=\{p\} \quad \text { or } \quad \operatorname{ker} L=\operatorname{Span}\left\{\psi_{\tau, \xi}^{j}, j=1,2\right\} \text { if } S=\{q\} .
$$

As for $P v_{\tau, \theta}$, we derive the expansions

$$
\begin{gathered}
P \psi_{\tau, \theta}^{0}=\psi_{\tau, \theta}^{0}-\frac{2}{\tau}+O\left(\rho_{n}^{2}\right) \\
P \psi_{\tau, \theta}^{j}=\psi_{\tau, \theta}^{j}+8 \pi(\beta+1) \frac{\partial H}{\partial \theta_{j}}(\cdot, \theta)+O\left(\rho_{n}^{2}\right), \quad j=1,2 .
\end{gathered}
$$

Since $\left\|P \psi_{\tau, \theta}^{0}\right\| \sim$ const., we have that the projection $P \psi_{\tau, \theta}^{0}$, up to renormalization, "almost" satisfies:

$$
-\Delta \frac{P \psi_{\tau, \theta}^{0}}{\left\|P \psi_{\tau, \theta}^{0}\right\|} \sim \rho_{n}^{2}|z-\theta|^{2 \beta} e^{v_{\tau, \theta}} \frac{P \psi_{\tau, \theta}^{0}}{\left\|P \psi_{\tau, \theta}^{0}\right\|}-\frac{2}{\tau\left\|P \psi_{\tau, \theta}^{0}\right\|} \rho_{n}^{2}|z-\theta|^{2 \beta} e^{v_{\tau, \theta}}
$$

and, in an "heuristic" way, we do not expect asymptotically the presence of $P \psi_{\tau, \theta}^{0}$ in the kernel of the linearized operator $L$ on $\Omega$. While, in case $S=\{q\}$ we have that

$$
-\Delta \frac{P \psi_{\tau, \xi}^{j}}{\left\|P \psi_{\tau, \xi}^{j}\right\|} \sim \rho_{n}^{2} e^{v_{\tau, \xi}} \frac{P \psi_{\tau, \xi}^{j}}{\left\|P \psi_{\tau, \xi}^{j}\right\|}+O\left(\rho_{n}^{3} e^{v_{\tau, \xi}}\right), j=1,2,
$$

since $\left\|P \psi_{\tau, \theta}^{j}\right\| \sim \frac{\text { const. }}{\rho_{n}}$ for $j=1,2$, and so we expect that the projection $P \psi_{\tau, \xi}^{j}$ is asymptotically in the kernel of $L$ in $\Omega^{\rho_{n}}$.

This is the key point of our construction, which displays a different feature of Liouville-type equations in dimension 2 with respect to analogous "critical problems" in higher dimensions.

For simplicity, we will omit in the sequel the apices and pedices. We consider the subspace of $H_{0}^{1}(\Omega)$ :

$$
K=\{0\} \text { if } S=\{p\} \quad \text { or } \quad K=\operatorname{Span}\left\{P \psi^{j}, \mathrm{~J}=1,2\right\} \text { if } S=\{q\},
$$

and we denote by $K^{\perp}$ its orthogonal space and $\Pi, \Pi^{\perp}$ the corresponding orthogonal projections. We introduce the linear operator $L_{n}$ defined as follows:

$$
L_{n}(\phi)= \begin{cases}\phi-i^{*}\left[\rho_{n}^{2}|z-p|^{2 \alpha} V(z) e^{P v} \phi\right] & \text { in case } S=\{p\} \\ \Pi^{\perp}\left\{\phi-i^{*}\left[\rho_{n}^{2} K(z) e^{P v} \phi\right]\right\} & \text { in case } S=\{q\},\end{cases}
$$

where $i^{*}$ is the adjoint operator of the immersion $H_{0}^{1}(\Omega) \hookrightarrow L^{\frac{p}{p-1}}(\Omega)$.

We have an invertibility property, similar in spirit to a result established in $\mathbb{R}^{2}$ by Chae-Imanuvilov in $[\mathrm{CI}]$ :

Proposition 8. There exists $N$ large and a constant $c>0$ such that, for any $n \geq N$ and $\xi$ in a compact subset of $\Omega^{\prime}=\Omega \cap\{K>0\}$, we have

$$
\left\|L_{n}(\phi)\right\| \leq \frac{c}{\left|\log \rho_{n}\right|}\|\phi\|, \quad \forall \phi \in K^{\perp} .
$$

In particular, the operator $L_{n}$ is invertible and $\left\|\left(L_{n}\right)^{-1}\right\| \leq \frac{\left|\log \rho_{n}\right|}{c}$.

The proof is by means of a contradiction argument, see for details [EGP]. Suppose there exist sequences

$$
\xi_{n} \rightarrow \xi \in \Omega^{\prime}, \phi_{n} \in K^{\perp}:\left\|\phi_{n}\right\|=1 \text { and }\left\|L_{n}\left(\phi_{n}\right)\right\|=o\left(\frac{1}{\left|\log \rho_{n}\right|}\right) .
$$


Via a rescaling argument, we can pass weakly to the limit by finding a non trivial solution $\phi_{0}$ for the linearized operator on $\mathbb{R}^{2}$ (whose kernel has dimension 1 in case $S=\{p\}$ and dimension 3 in case $S=\{q\})$. In the limit, the property $\phi_{n} \in K^{\perp}$ gives that $\phi_{0}$ is orthogonal to $\psi_{1,0}^{j}, j=1,2$, in case $S=\{q\}$. Once we are able to show that $\phi_{0}$ gains automatically the orthogonality with respect to $\psi_{1,0}^{0}$, we reach the contradiction $\phi_{0}=0$.

In order to do this, we construct a test function $u_{0}$ such that

$$
-\Delta u_{0}-\rho_{n}^{2}|z-\theta|^{2 \beta} e^{v} u_{0} \sim \rho_{n}^{2}|z-\theta|^{2 \beta} e^{v} \psi^{0},\left\|u_{0}\right\| \sim \text { const. }\left|\log \rho_{n}\right| .
$$

The first relation indicates that $P \psi^{0}$, which corresponds in the duality with the R.H.S., is "essentially" in the range of $L_{n}$. The second relation implies that the inverse operator of $L_{n}$, if it exists, has norm which explodes exactely as $\left|\log \rho_{n}\right|$.

Multiplying the equation satisfied by $\phi_{n}$ for $P u_{0}$ and passing to the limit, we obtain the third orthogonality relation.

In order to define the test function $u_{0}$, let us consider

$$
\begin{aligned}
u_{\tau, \theta}= & \frac{4}{3 \tau} \log \left(\tau^{2} \rho_{n}^{2}+|z-\theta|^{2(1+\beta)}\right) \frac{\tau^{2} \rho_{n}^{2}-|z-\theta|^{2(1+\beta)}}{\tau^{2} \rho_{n}^{2}+|z-\theta|^{2(1+\beta)}}+\frac{8}{3} \frac{\tau \rho_{n}^{2}}{\tau^{2} \rho_{n}^{2}+|z-\theta|^{2(1+\beta)}} \\
& -\frac{32}{3} \pi(\beta+1) H(\theta, \theta) \frac{\tau \rho_{n}^{2}}{\tau^{2} \rho_{n}^{2}+|z-\theta|^{2(1+\beta)}} .
\end{aligned}
$$

Now, define $u_{0}$ in the following form:

$$
u_{0}=P u_{\tau(p), p} \text { if } S=\{p\} \quad \text { or } \quad u_{0}=P u_{\tau\left(\xi_{n}\right), \xi_{n}} \text { if } S=\{q\} .
$$

The first two terms in (13) are obtained simply by solving the equation in (12) in the radial case. However, to adjust their boundary values by projecting in $H_{0}^{1}(\Omega)$, one creates an additional term which we control by adding the third term in (13).

In case $S=\{p\}$, by Proposition 8 and a fixed point argument, we find that for $n$ large there exists $\phi_{n} \in K^{\perp}=H_{0}^{1}(\Omega)$ such that $\left\|\phi_{n}\right\| \rightarrow_{n \rightarrow+\infty} 0$ and $P v+\phi_{n}-i^{*}\left(\rho_{n}^{2}|z-p|^{2 \alpha} V(z) e^{P v+\phi_{n}}\right)=0$. Hence, we obtain a solution sequence for (5) in the form $P v_{\tau(p), p}+\phi_{n}$ concentrating in $p$.

In case $S=\{q\}$, in a similar way as above, we find that, for $n$ large and for any $\xi$ in a compact subset of $\Omega^{\prime}=\Omega \cap\{K>0\}$, there exists $\phi_{n}=\phi_{n}(\xi) \in K^{\perp}$ such that $\left\|\phi_{n}\right\| \rightarrow_{n \rightarrow+\infty} 0$ uniformly in $\xi$ and

$$
\Pi^{\perp}\left[P v+\phi_{n}-i^{*}\left(\rho^{2} K(z) e^{P v+\phi_{n}}\right)\right]=0 .
$$

At this point, it is possible to reduce the problem to a finite dimensional. Let us define the energy functional

$$
E_{n}(u)=\frac{1}{2} \int_{\Omega}|\nabla u|^{2}-\rho_{n}^{2} \int_{\Omega} K(z) e^{u}, u \in H_{0}^{1}(\Omega),
$$

whose critical points correspond to solutions of (5). We have the following property:

$$
\nabla E_{n}\left(P v+\phi_{n}(\xi)\right)=0 \text { iff } \xi \text { is a c.p. of } \xi \rightarrow E_{n}\left(P v+\phi_{n}(\xi)\right),
$$

which reduces the search of solutions to find critical points for some finite dimensional functional. We can perform an asymptotic expansion for the reduced functional and get

$$
E_{n}\left(P v+\phi_{n}(\xi)\right)=\left(-16 \pi \log \rho_{n}+24 \pi \log 2-16 \pi\right)-32 \pi^{2} \mathcal{F}(\xi)+o(1)
$$

as $n \rightarrow+\infty C^{1}$-uniformly on compact sets of $\Omega^{\prime}$. This guarantees that a "stable" critical point $q$ of $\mathcal{F}$ generates a solution sequence for (5) concentrating there.

In particular, we get the following result:

Theorem 9. [CL3] For any domain $\Omega$ there exists a solution sequence of (5) concentrating at a minimum point of $H(\xi, \xi)+\frac{1}{4 \pi} \log K(\xi)$. 


\section{References}

[Au] T. Aubin, Some Nonlinear Problems in Riemannian Geometry, Springer-Verlag, Berlin Heidelberg, New York, 1998.

[Ba] A. Bahri, Critical point at infinity in some variational problems, Pitman Research Notes Math. 182, Longman House, Harlow (1989).

[BP] S. Baraket, F. Pacard, Construction of singular limits for a semilinear elliptic equation in dimension 2, Calc. Var. Partial Differential Equations 6 (1998), 1-38.

[BCLT] D. Bartolucci, C.C. Chen, C.S. Lin, G. Tarantello, Profile of blow-up solutions to mean field equations with singular data, Comm. Partial Differential Equations, to appear.

[BT1] D. Bartolucci, G. Tarantello, The Liouville equation with singular data: a concentrationcompactness principle via a local representation formula, J. Differential Equations 185 (2002), 161-180.

[BT2] D. Bartolucci, G. Tarantello, Liouville type equations with singular data and their applications to periodic multivortices for the electroweak theory, Comm. Math. Phys. 229 (2002), 3-47.

[BBH] F. Bethuel, H. Brezis, F. Helein, Ginzburg Landau Vortices, Birkhauser, 1994.

$[\mathrm{BM}] \mathrm{H}$. Brezis, F. Merle, Uniform estimates and blow-up behavior for solutions of $-\Delta u=V(x) e^{u}$ in two dimensions, Comm. Partial Differential Equations 16 (1991), 1223-1253.

[CHS] L. A. Caffarelli, R. Hardt, L. Simon, Minimal surfaces with isolated singularities, Manuscripta Math. 48 (1984), 1-18.

[CLMP1] E. Caglioti, P.L. Lions, C. Marchioro, M. Pulvirenti, A special class of stationary flows for two-dimensional Euler equations: a statistical mechanics description, Comm. Math. Phys. 143 (1992), 501-525.

[CLMP2] E. Caglioti, P.L. Lions, C. Marchioro, M. Pulvirenti, A special class of stationary flows for two-dimensional Euler equations: a statistical mechanics description Part II, Comm. Math. Phys. 174 (1995), 229-260.

[CI] D. Chae, O. Imanuvilov, The existence of non-topological multivortex solutions in the relativistic self-dual Chern-Simons theory, Comm. Math. Phys. 215 (2000), 119-142.

[CLi] W. Chen, C. Li, Classification of solutions of some nonlinear elliptic equations, Duke Math. J. 63 (1991), 615-623.

[CL3] C.C. Chen, C.S. Lin, On the simmetry of blowup solutions to a mean field equation, Ann. Inst. H. Poincaré Anal. Non Linéaire 18 (2001), 271-296.

[CW] K.S. Chou, T.Y.H. Wan, Asymptotic radial symmetry for solutions of $\triangle u+\exp u$ in a punctured disc, Pacific J. of Math. 163 (1994), 269-276.

[CR] M.G. Crandall, P.H. Rabinowitz, Some continuation and variational methods for positive solutions of nonlinear elliptic eigenvalue problems, Arch. Ration. Mech. Anal. 58 (1975), 207-218.

[Es1] P. Esposito, Blow up solutions for a Liouville equation with singular data, SIAM J. Math. Anal., to appear.

[Es2] P. Esposito, A class of Liouville-type equations arising in Chern-Simons vortex theory: asymptotics and construction of blowing up solutions, Thesis, Roma "Tor Vergata", 2003. 
[EGP] P. Esposito, M. Grossi, A. Pistoia, On the existence of blowing-up solutions for a mean field equation, Ann. Inst. H. Poincaré Anal. Non Linéaire, to appear.

[GNN] B. Gidas, W.M. Ni, L. Nirenberg, Symmetry and related properties via the maximum principle, Comm. Math. Phys. 68 (1979), 209-243.

[GT] D. Gilbarg, N. S. Trudinger, Elliptic partial differential equations of second order, 2nd Edition, Springer Verlag, 1983.

[HKP] J. Hong, Y. Kim, P.Y. Pac, Multivortex solutions of the Abelian Chern-Simons theory, Phys. Rev. Lett. 64 (1990), 2230-2233.

[JW] R. Jackiw, E. Weinberg, Self-dual Chern-Simons vortices, Phys. Rev. Lett. 64 (1990), 2234-2237.

[Lio] J. Liouville, Sur l'équation aud dérivées partielles $\partial^{2} \log \lambda / \partial u \partial v \pm 2 \lambda a^{2}=0$, J. de Math. 18 (1853), 71-72.

[MW] L. Ma, J. Wei, Convergence for a Liouville equation, Comment. Math. Helv. 76 (2001), 506-514.

[Po] S. Pohozaev, Eigenfunctions of the equation $\Delta u+\lambda f(u)=0$, Soviet. Math. Dokl. 6 (1965), 1408-1411.

[PT] J. Prajapat, G. Tarantello, On a class of elliptic problem in $\mathbb{R}^{2}$ : symmetry and uniqueness results, Proc. Roy. Soc. Edinburgh Sect. A 131 (2001), 967-985.

[Re] O. Rey, The role of Green's function in a nonlinear elliptic equation involving the critical Sobolev exponent, J. Funct. Anal. 89 (1990), 1-52.

[Su] T. Suzuki, Two dimensional Emden-Fowler equation with exponential nonlinearity, Nonlinear Diffusion Equations and their equilibrium states 3 (1992), 493-512.

[Ta1] G. Tarantello, A quantization property for blow up solutions of singular Liouville-type equations, J. Funct. Anal., to appear.

[Ta2] G. Tarantello, An Harnack inequality for Liouville-type equations with singular sources, Indiana Univ. Math. J., to appear.

[Ta3] G. Tarantello, Analytical aspects of Liouville-type equations with singular sources, Handbook of Differential Equations (M. Chipot, P.Quitter ed.), Elsevier, North Holland, to appear.

[Ta] C. Taubes, Arbitrary N-vortex solutions for the first order Ginzburg-Landau equations, Comm. Math. Phys. 72 (1980), 277-292.

[Ya] Y. Yang, Solitons in field theory and nonlinear analysis, Springer Verlag, 2001. 